
\title{
S Research Square \\ Treatment of Facial Nevi with Ideal Design and Splitting Technique
}

\section{Qiang Sun (D261142065@qq.com)}

The First Hospital of China Medical University: The First Affiliated Hospital of China Medical University https://orcid.org/0000-0001-7659-9708

\section{Jiayan Guo}

The First Hospital of China Medical University: The First Affiliated Hospital of China Medical University

\section{Siyu Liu}

The First Hospital of China Medical University: The First Affiliated Hospital of China Medical University

\section{Mengru Zhu}

The First Hospital of China Medical University: The First Affiliated Hospital of China Medical University Jingyi Feng

The First Hospital of China Medical University: The First Affiliated Hospital of China Medical University

\section{You Zhou}

The First Hospital of China Medical University: The First Affiliated Hospital of China Medical University

\section{Yuxin Wang}

The First Hospital of China Medical University: The First Affiliated Hospital of China Medical University Shu Guo

The First Hospital of China Medical University: The First Affiliated Hospital of China Medical University

\section{Research article}

Keywords: Splitting technique, Facial nevus

Posted Date: March 22nd, 2021

DOI: https://doi.org/10.21203/rs.3.rs-330100/v1

License: (c) (1) This work is licensed under a Creative Commons Attribution 4.0 International License. Read Full License 


\section{Abstract}

Background: The treatment of nevi includes surgical treatment and non-surgical treatment. Non-surgical treatment has many defects in the clinic, whereas surgical treatment is applicable to any type of nevi. However, there is no unified standard for surgical methods.

Methods: Patients with facial nevi ( width $\leq 4 \mathrm{~cm}$ ) and high requirements for beauty were included. Preoperatively, incision design and resection range based on the recommended wrinkles or folds of each region and principle of plastic surgery. Intraoperatively, a nevus flap was formed, and then equally divided by splitting technique. After the splitting nevus flap was resected, suture without tension was performed.

Results: 21 patients underwent surgical excision. 14 patients underwent complete excision, while 7 patients underwent serial excision. The patients were satisfied with the appearance, local sensations were normal, and there were no secondary deformities of the surrounding facial organs without recurrence.

Conclusion: This method is of added value, which achieved by the correct assessment of the size and location of facial nevi and designed according to the reference marks in each region and complete or serial excision.

\section{Background}

Skin nevi are common benign diseases, which generally do not affect the patient's physical health and quality of life. In the past, only when the nevus suddenly became large, inflammation, bleeding, itchy, or sclerosis and/or small satellite nevi appeared, treatment would be implemented. However, with the improvement of living standards, people's requirements and investment in beauty have increased annually. Nevi on the face affects beauty and psychology, thereby, the number of patients who require treatment also increases year after year. At the same time, in order to avoid the complication of the original simple surgery and delaying the optimal treatment opportunity, the age of patients requiring for treatment gradually declines $(1,2)$.

There are many methods to treat nevi, which can be divided into non-surgical and surgical treatments. Non-surgical treatment mainly includes chemical corrosion, cryotherapy, electrolysis, high-frequency electro tome, laser, etc., which destroys and sheds the lesion, and repairs the defect by surrounding tissue. Although surgery can be avoided, the depth of tissue destruction is not easy to control. If it is too shallow, the treatment is unthorough, and easy to irritate the lesion. If it is too deep, it will leave a depressed scar and local skin pigmentation $(3,4)$. Surgical treatment is applicable to any type of nevus, regardless of the size (5). It mainly includes direct excision, serial excision, skin graft, skin flap transplantation, and the use of tissue expander $(6,7)$. As we know, the concept of surgical treatment should be about using the simplest method with the least pain, to get the slightest, concealed and not ugly scar without disfigurement. The objective of this report is to share the author 's experience with ideal design and splitting technique to treat facial nevi. 


\section{Patients And Methods}

\section{Patient group}

All these 21 patients were selected from plastic surgery in the first hospital of China Medical University between March 2018 and December 2019. There are 6 male and 15 female patients. Patients' ages ranged from 1 to 26 , with an average of 6.3 years old. 3 cases were on the forehead, 2 cases were on the nasal dorsum, 5 cases were on the cheek, the other 5 cases were on the outside of the orbit, 4 cases were on the lower eyelid, and 2 cases are in the region near nose and lips.

Inclusion criteria: Patients with high requirements for beauty, and small or medium size benign nevi on the face, no limitation in length, the width $\leq 4 \mathrm{~cm}$.

Exclusion criteria: 1 . The nevus may be malignant, which should be removed or enlarged resection in time, according to the history and clinical manifestation: sudden increase in size, darker color, ulcer, swelling, repeated infection, painful, itchy, or the emergence of satellite lesions (8). 2 . The width of the nevus is $₫ 4 \mathrm{~cm}$.

\section{Surgical technique}

Incision design: The incision should be at least $1 \mathrm{~mm}$ outside the edge of the lesion. The principle of the axial direction (suture direction): if wrinkles or skin lines are obvious, choose the direction along the wrinkle or skin line as much as possible; if not, along Langer's line (Fig.1, Fig.2A, Fig.3A). Partition boundaries, facial organ contours, and other hidden lines are also good choices. However, if the aspect ratio of the nevus is $₫ 3: 1$, the incision can be along the major axis of the nevus, which may be inconsistent with the above principle.

Resection range: Generally, the resection amount each time should be maximized to reduce the number of operations, which is determined according to the degree of local skin tension, so as not to cause obvious deformation and displacement of adjacent tissue and organ. If the nevus is removed in stages, a fusiform incision should be made in the middle part of nevus, not exceeding the range of the nevus as far as possible, for the first time (Fig.2A).

Surgery method: Surgical area is widely injected with a large amount of $0.5 \%$ lidocaine containing 1:200,000 epinephrine, especially on the side far away from important organs (Fig.2A). The first incision should be performed on the side adjacent to important organs. Then, using the other side as the pedicle, the nevus flap is formed by extensive and sufficient subcutaneous dissection, which contained superficial fat (Fig.2B). The flap is cut into two aliquots along the middle line of the flap until reaching the designed incision. Make a subcutaneous suture between the two flaps with a 4-0 coated Vicryl suture; at the same time, observe the local tension and ensure whether the important organ is displaced. With the same method, the newly formed flaps are equally divided and sutured (Fig.2C). The number of splitting flaps is according to the size of nevi. After the incision is divided equally, the small nevus flaps are removed, and the intermittent suture is performed in the subcutaneous and intradermal layers with 4-0 and 5-0 
coated Vicryl suture respectively (Fig.2D). At last, 4-0 or 6-0 nitinol suture is used to make continuous intradermal suture to tightly close the incision in the absence of tension.

Postoperative treatment: Nevi needed to be pathologically examined after surgery. The suture was taken out 7-21 days after surgery. Serial excision was performed at least 3-6 months after surgery (Fig.2E,F).

\section{Results}

14 patients underwent direct complete excision (Fig.3); 7 patients underwent serial excision, and two surgeries were enough for complete excision (Fig.2). Postoperative pathological reports: 8 cases were junctional nevi, 10 cases were compound nevi, 2 cases were intradermal nevi, and 1 case was sebaceous nevus. All the patients were treated with topical drugs to prevent scar hyperplasia after surgery. The follow-up period was 6-15 months, and there was no sign of recurrence. The appearance was satisfactory, and there were no secondary deformities of the surrounding facial organs.

\section{Discussion}

The position and size of facial nevi are the two main factors that influence the design of the surgical method and the postoperative effect. Some scholars have classified nevi according to their size: small (<1.5 cm); medium (M1: 1.5-10 cm, M2:> 10-20 cm); large (L1:> 20-30 cm, L2 :> 30-40 cm); and giant (G1:> 40-60 cm, G2:> $60 \mathrm{~cm}$ ) (8). Obviously, this classification is not suitable for guiding the surgical treatment of facial nevi (9). In the specific operation, it was found that the size of facial nevi was a secondary consideration compared to the facial aesthetic unit in which it was located (10). The majority of patients in this group were children under 3 years old. For children, the face did not have obvious wrinkles, so the surgeon was required to be proficient in the distribution and direction of wrinkles on the face. We divided the face into 11 units referring to the distribution and direction of wrinkles or skin lines, and clarified the reference marks for designing the axial direction of the incision in each unit, which had great reference value, especially for children. For the repair of lesions across units, this principle must also be followed.

Although we have comprehensive reference marks, but not all incisions can be designed according to the above principle(10). If the aspect ratio of the nevus is $₫ 3: 1$, and the direction of the major axis of the nevus is inconsistent with the above principle, it is more difficult to design an ideal incision line. In this case, it is more common practice to design the incision along the major axis of the nevus (Fig.3). However, we can change the direction of nevus through the method of serial excision. Sometimes the purpose of serial excision is only to change the direction of the long axis of nevus (Fig.2). Whereas, if the difference between length and width is not obvious, direct excision requires prolonged incision to prevent the formation of "dog ear" and long scar. Even though patients are hard to accept serial excision, better long-term results make it possible $(11,12)$. After 3 to 6 months, the scar is basically softened, and the skin tissue is naturally relaxed. And then, a further excision is performed until the whole nevus are removed. 
After satisfying above-mentioned principles, reducing the number of operations becomes the primary issue. Extensive, massive injection of anesthetics can expand the skin tissue immediately during the operation. The nevus flap with extensive and sufficient subcutaneous dissection can ensure more resection of the lesion tissue and avoid the displacement of important organs. In the past, we usually gripped the skin tissue along the major axis of the nevus to judge the amount of the nevus that can be removed by experience. Under splitting technique, the nevus flap is cut perpendicularly until it reaches the designed incision in the absence of tension, thereby, delimiting the maximum amount of tissue which can be removed, which is important for serial resection. Meanwhile, this technique also helps to disperse and reduce tension. The nevus flap is cut along its own middle line, but the suture does not need to be perpendicular to the major axis of the nevus as usual. Less tension means the facial organ is less likely to be displaced. Thus, it is the golden standard of how to suture. After using this technique, the amount of removed lesion is often much more than designed amount before surgery (Fig.3), and nevi in our group were resected at most twice. Because the nevus flap is cut equally, there is less "dog-ear", and the incision is shortened. Finally, continuous intradermal suture with nitinol suture is used to tightly close the incision, which can avoid skin surface suture to irritate and cut skin, and extend stitch removal time to 3 weeks after surgery depending on the specific situation. Long-term tension reduction will help incision healing and inhibit scar widening.

In order to prevent nevus cell planting during surgery, several aspects should be paid attention : 1 . Surgical blades, scissors, tweezers and other instruments that have contacted the nevus cells should no longer contact normal skin and subcutaneous adipose tissue. 2 . The dissection should be performed in deep subcutaneous tissue, but not the superficial layer(13-15). This group did not find any seeding of nevus cells due to surgery.

\section{Conclusion}

The treatment of facial nevi should be determined by comprehensive consideration of various factors. Better cosmetic results can be achieved by the correct assessment of the size, aspect ratio and location of facial nevi, the ideal design of the axial direction of the incision according to the reference marks in each unit, and the application of splitting technique. Our method is suitable for facial nevi, of which the width $\leq 4 \mathrm{~cm}$. For the width $>4.0 \mathrm{~m}$, other methods such as skin expanders or full-thickness skin grafts can be used. Also, more cases are needed to prove it.

\section{Declarations}

\section{Ethics Approval and consent to participate}

The study was approved by ethical review board of China Medical University, which followed the ethical principles of the Declaration of Helsinki 1964, and all the participants (or their parent or legal guardian in the case of children under 18) provided the written informed consent. 


\section{Consent to publish}

Written consent for publication were obtained from participants (or their parent or legal guardian in the case of children under 18) for information and medical images about the participant himself/herself.

\section{Competing Interests}

The authors have declared that they have no conflict of interest.

\section{Availability of data and materials}

The datasets used and analyzed during the current study are available from the corresponding author on reasonable request.

\section{Funding}

None.

\section{Authors' contributions}

SQ and LSY designed this study. FJY, GJY and ZY collected literatures and conducted the analysis of data. WYX, GS and ZMR drafted the manuscript. SQ and LSY wrote the manuscript. All authors contributed to review the manuscript. All authors read and approved the final manuscript.

\section{Acknowledgement}

We appreciate the help from Ziwei Zhang in The First Hospital of China Medical University.

\section{References}

1. Lee H, Eom Y, Oh KS. Management of Congenital Melanocytic Nevus on Face Using Multiple Reexpansion Method: Aesthetic and Psychosocial Results. J Craniofac Surg. 2019;30(8):2385-9.

2. Captier G. [The congenital melanocytic nevi of the face in child: What's new?]. Rev Stomatol Chir Maxillofac Chir Orale. 2015;116(4):187-99.

3. Meesters AA, Wind BS, Kroon MW, Wolkerstorfer A, et al. Ablative fractional laser therapy as treatment for Becker nevus: a randomized controlled pilot study. Journal of the American Academy of Dermatology. 2011;65(6):1173-9.

4. Langrock ML, Hohenleutner U. Multiple recurrences of an earlobe keloid: treatments with carbon dioxide laser vaporization, cryotherapy and finally keloid resection. J Dtsch Dermatol Ges. 2009;7(6):560-2. 
5. Almutairi HM, Al-hothali GI. The outcome of using different surgical modalities and laser therapy in the treatment of small- and medium-sized congenital melanocytic nevi: a systematic review. International Journal of Dermatology. 2019;n/a(n/a).

6. Hassanein AH, Rogers GF, Greene AK. Management of challenging congenital melanocytic nevi: outcomes study of serial excision. J Pediatr Surg. 2015;50(4):613-6.

7. Carneiro Junior LVdF, Aguiar LFdS, Pitanguy I. Tratamento cirúrgico do nevo melanocítico gigante. Revista Brasileira de Cirurgia Plástica. 2011;26(2):198-204.

8. Krengel S, Scope A, Dusza SW, Vonthein R, et al. New recommendations for the categorization of cutaneous features of congenital melanocytic nevi. Journal of the American Academy of Dermatology. 2013;68(3):441-51.

9. Ott $H$, Krengel $S$, Beck $O$, Bohler $K$, et al. Multidisciplinary long-term care and modern surgical treatment of congenital melanocytic nevi - recommendations by the CMN surgery network. Journal der Deutschen Dermatologischen Gesellschaft = Journal of the German Society of Dermatology : JDDG. 2019;17(10):1005-16.

10. Fattahi TT. An overview of facial aesthetic units. Journal of oral and maxillofacial surgery : official journal of the American Association of Oral and Maxillofacial Surgeons. 2003;61(10):1207-11.

11. Li X, Wang T, Li W, Guo X, et al. [Surgical treatment of eyelid divided nevus]. Zhonghua zheng xing wai ke za zhi $=$ Zhonghua zhengxing waike zazhi $=$ Chinese journal of plastic surgery. 2015;31(2):969.

12. Su JJ, Chang DK, Mailey B, Gosman A. Treatment of a giant congenital melanocytic nevus in the adult: review of the current management of giant congenital melanocytic nevus. Ann Plast Surg. 2015;74 Suppl 1:S57-61.

13. Rosa RM, Caiado RC, Reis PR, Lacerda Ede P, et al. Influence of surgical technique in the peritoneal carcinomatosis surgical wound implant: experimental model in mice. Arquivos brasileiros de cirurgia digestiva : $A B C D=$ Brazilian archives of digestive surgery. 2015;28(1):13-6.

14. Engilbertsson $\mathrm{H}$, Aaltonen KE, Bjornsson S, Kristmundsson T, et al. Transurethral bladder tumor resection can cause seeding of cancer cells into the bloodstream. The Journal of urology. 2015;193(1):53-7.

15. Wu JS, Guo LW, Ruiz MB, Pfister SM, et al. Excision of trocar sites reduces tumor implantation in an animal model. Diseases of the colon and rectum. 1998;41(9):1107-11.

\section{Figures}




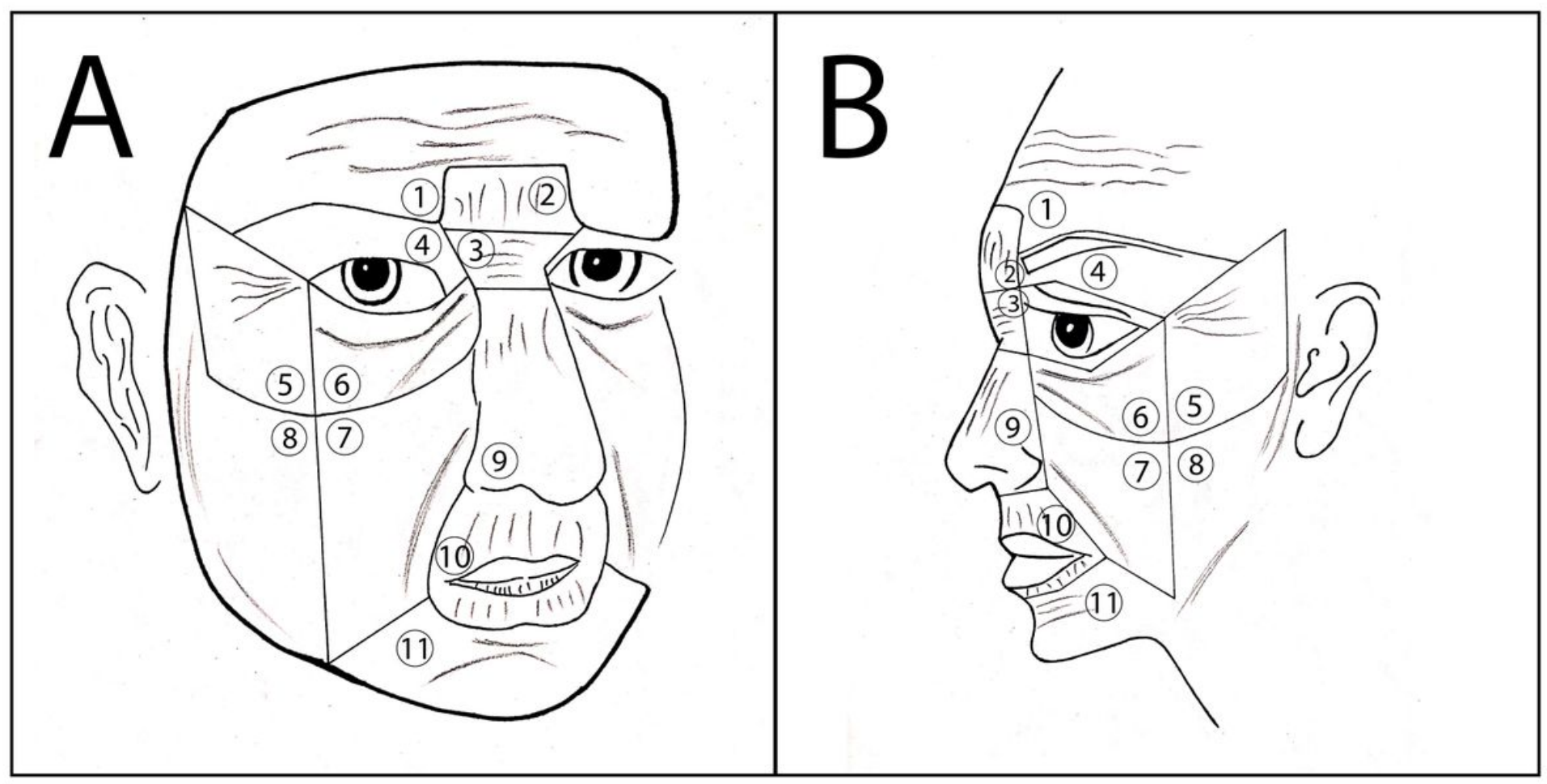

\section{FIGURE 1}

\section{Figure 1}

. The facial regions for designing incision. The face is divided into 11 regions based on the main wrinkles or folds for each regions: $₫$ forehead: horizontal frontal lines; 『intercilium: vertical lines; $₫$ nasal root: horizontal nasal root lines; \upper eyelid: lines parallel to the eyelid margin or double eyelid line; \outside of the orbit: crow's feet; 『lower eyelid: parallel to the eyelid margin, the tear trough or palpebromalar groove; 邓region near to nose and lips: oblique nasolabial fold; खcheek (side): vertical lines nearly parallel to the sidebum or folds in the front of ears; 『nasal dorsum: longitudinal lines; \upper and lower lips: longitudinal lip lines; 『chin: mentolabial sulcus. 


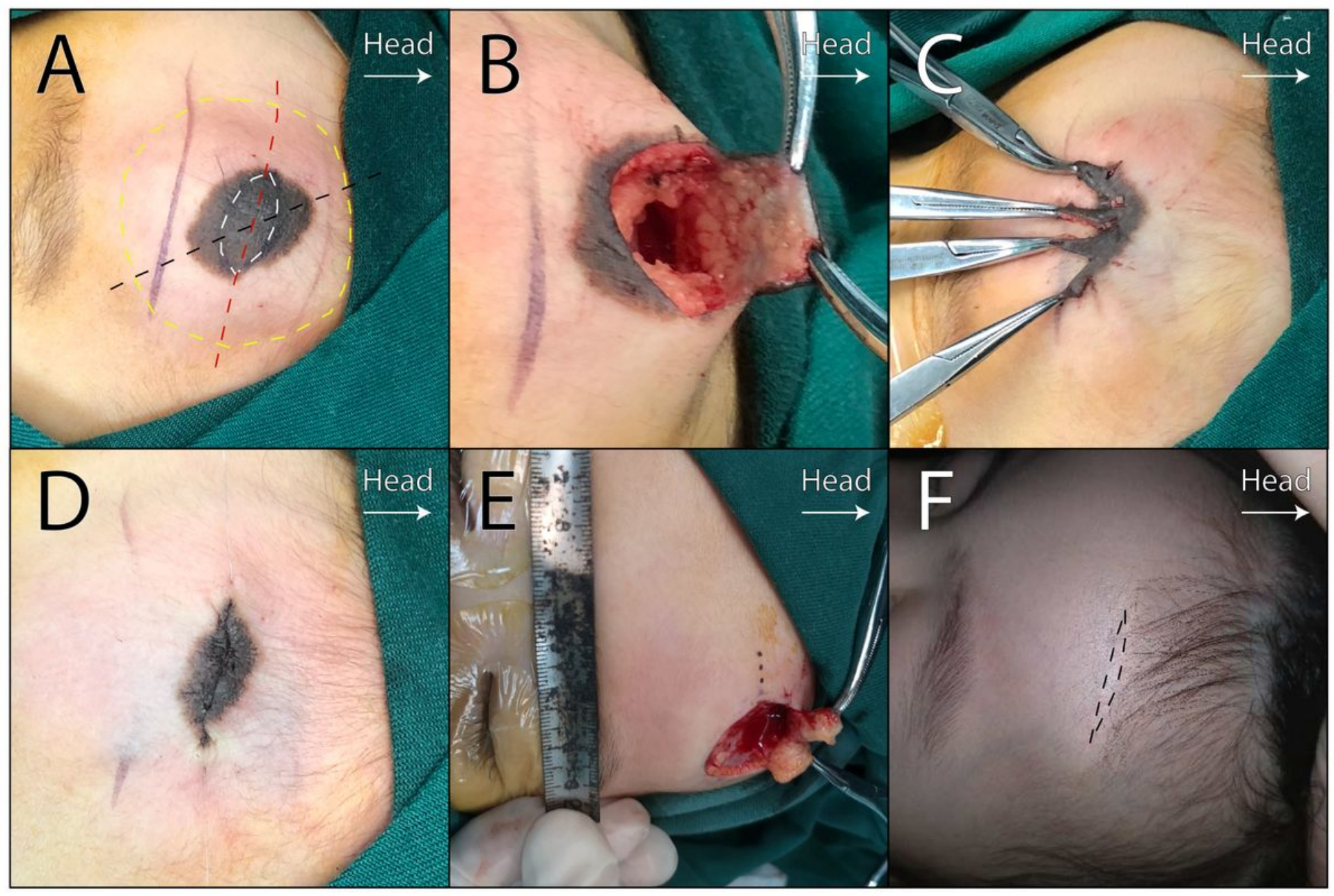

Figure 2

A patient with compound nevus on the left forehead underwent serial excision. (A) The nevus was 3.0 $\mathrm{cm} \times 2.0 \mathrm{~cm}$, and the black dashed line was the major axis of the nevus, which is not along forehead wrinkles and hairline. The major axis of the nevus was changed to the red dashed line which was along forehead wrinkles, and the incision was designed as the white line. 0.5\% lidocaine containing 1:200,000 epinephrine was injected in the area within the yellow dashed line. (B) The first incision was performed on the side adjacent to the left eyebrow and eye, and extensive and sufficient subcutaneous dissection on the other side. (C) The nevus flap is cut into four aliquots until reaching the designed incision. (D) The small nevus flaps are removed, and the intermittent suture is performed in the subcutaneous and intradermal layers. (E) Serial excision was performed in the 6th month after surgery. (F) The appearance was satisfactory, and there were no secondary deformities of the surrounding facial organs in the 6th month after last surgery. 


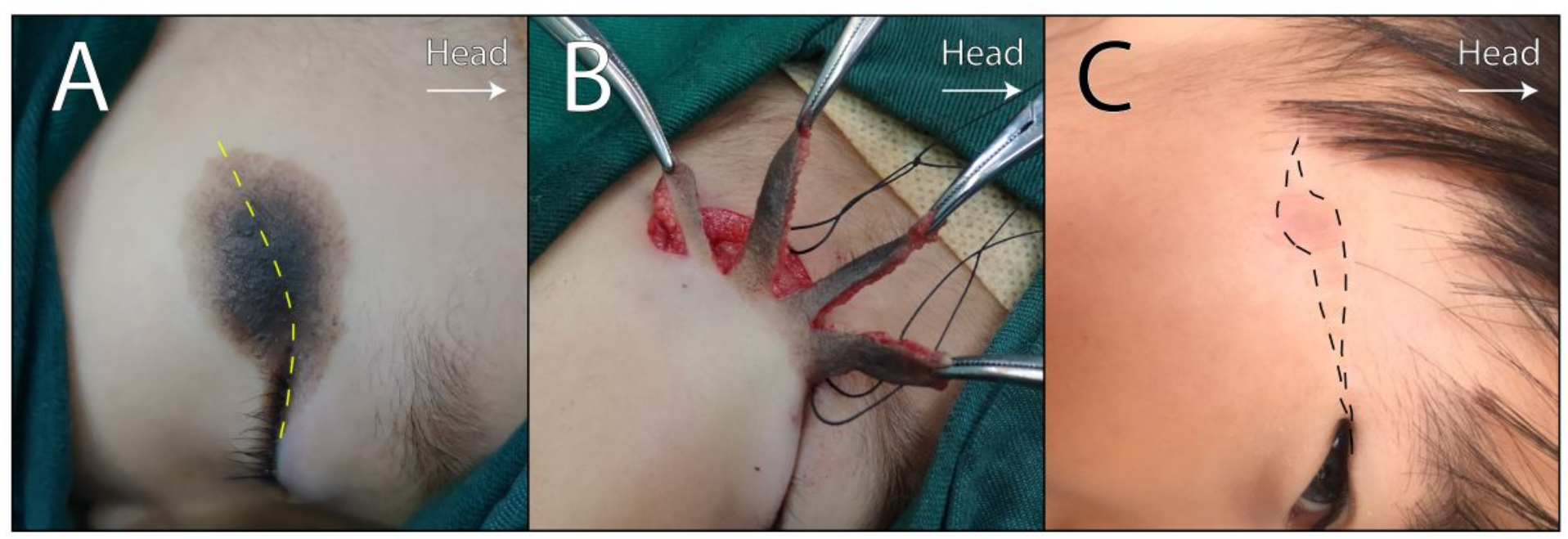

\section{Figure 3}

A patient with compound nevus on the right lateral orbital region underwent serial excision. (A) The nevus was $4.0 \mathrm{~cm} \times 2.5 \mathrm{~cm}$, and the major axis of the nevus was the yellow dashed line along the crow's feet. (B) The nevus flap was cut into four aliquots until reaching at least $1 \mathrm{~mm}$ outside the edge of the nevus. (C) The appearance was satisfactory, and there were no secondary deformities of the surrounding facial organs in the 15th month after last surgery. 